\title{
On the fabrication of micro- and nano-sized objects: the role of interstitial clusters
}

\author{
Diego R. Gomes ${ }^{1}$, Anatoliy A. Turkin ${ }^{1,2}$, David I. Vainchtein ${ }^{1}$, and Jeff Th. M. De Hosson ${ }^{1, *}$ \\ ${ }^{1}$ Department of Applied Physics, Zernike Institute for Advanced Materials, University of Groningen, Nijenborgh 4, \\ 9747 AG Groningen, The Netherlands \\ ${ }^{2}$ National Science Center "Kharkiv Institute of Physics and Technology", Akademichna St. 1, Kharkiv 61108, Ukraine
}

Received: 17 December 2017

Accepted: 23 January 2018

Published online:

1 February 2018

(C) The Author(s) 2018. This article is an open access publication

\begin{abstract}
Ion-induced bending phenomena were studied in free-standing nano-sized $\mathrm{Al}$ cantilevers with thicknesses in the range of $89-200 \mathrm{~nm}$. The objective is to present a predictive and useful model for the fabrication of micro- and nanosized specimens. Samples were irradiated in a Tescan Lyra dual beam system with $30 \mathrm{kV} \mathrm{Ga}^{+}$ions normal to the sample surface up to a maximum fluence of $\sim 2 \times 10^{21} \mathrm{~m}^{-2}$. Irrespective of thickness, all samples bent initially away from the $\mathrm{Ga}^{+}$beam; as irradiation proceeded, the bending direction was reversed. The $\mathrm{Al}$ cantilever bending behavior is discussed in terms of depth-dependent volume change due to implanted Ga atoms, radiation-induced point defects and interstitial clusters. A kinetic model is designed which is based on a set of rate equations for concentrations of vacancies, interstitial atoms, Ga atoms and clusters of interstitial atoms. The bending crossover is explained by the formation of sessile interstitial clusters in a zone beyond the $\mathrm{Ga}^{+}$penetration range. Model predictions agree with our experimental findings.
\end{abstract}

\section{Introduction}

Focused ion beams are used in the fabrication of micro- and nano-sized products [1-4]. A rather recent application, which is the topic of this contribution, is the bending of free-standing thin structures such as films, nanotubes and nanowires [5-11]. The mechanisms controlling this phenomenon are not sufficiently clear in a quantitative way, and different ideas have been proposed but rather qualitatively [11-13]. The production of crystallographic defects due to ion irradiation is widely investigated and well understood, with the collision cascade model being the most accepted. Nevertheless, the small sizes of the introduced features (point defects (PD) and their clusters of atomic dimensions up to few nanometers) limit the possibility of a comprehensive description by direct observation. As a result, molecular dynamics (MD) calculations are used to simulate cascades at a high level of sophistication [14, 15]. These are, however, usually limited by the available processing capacity to small volumes or number of consecutive cascades-full simulation of a bending experiment in a $5 \times 2 \times 0.2 \mu^{3}$ cantilever would

Address correspondence to E-mail: j.t.m.de.hosson@rug.nl 
involve, for instance, the order of $10^{10}$ cascade events with about $1.2 \times 10^{11}$ atoms each, making it unpractical at the present time.

Literature on ion-induced bending reports that the irradiated structures bend most of the time toward the incident beam. Yoshida et al. [11] reported bending away from the beam when the ion accelerating voltage was increased so that the implantation region would fall on the opposite side of the cantilever's neutral axis. The motivation for this paper was a rather unexpected and surprising observation made on aluminum cantilevers, which exhibited both bending away from the beam under irradiation with $30 \mathrm{kV}$ Ga ions, and a reversal of bending direction as irradiation progressed. This is a quite interesting observations from a fundamental viewpoint but also for the field of applications of nano- and microsized objects.

Recent experiments confirmed formation of a dislocation network [16] in Au nanoparticles and dislocation loops in the Al thin film [17] at fluences typical for focused ion beam (FIB) milling. TEM observation of as-fabricated $\mathrm{Al}$ nanopillars revealed the formation of nm-sized dislocation loops [18]. According to [17], the dislocation loops formed by a high energy $\mathrm{Ga}^{+}$ ion impact in $\mathrm{Al}$ at room temperature are most likely of the interstitial type.

Here, we propose a kinetic model based on the diffusion of glissile interstitial clusters from the cascade zone to the non-irradiated zone. The estimations of this model were compared to experiments in aluminum cantilevers with a thickness varying from 90 to $200 \mathrm{~nm}$ with good agreement. The ability to accurately predict and control the deflection of nanostructures may turn the FIB into an important tool in the design and fabrication of miniaturized devices.

\section{Experiments and results}

Films of aluminum with nominal thicknesses of 200, 144 and $89 \mathrm{~nm}$ were deposited on mechanically polished $\mathrm{NaCl}$ substrates using a Temescal FC-2000 electron-beam evaporator at a vacuum of $8 \times 10^{-7}$ Torr and an evaporation rate of $0.1 \mathrm{~nm} \mathrm{~s}^{-1}$. The films were made free-standing by dissolving the substrate in distilled water and collecting the floating films with a TEM grid. The samples were annealed at $200{ }^{\circ} \mathrm{C}$ for $30 \mathrm{~min}$ in argon atmosphere and then mounted in a home-designed holder that enables the grid surface to be positioned normally to the incident ion beam in a Tescan Lyra FIB-SEM dual system.

An ion acceleration voltage of $30 \mathrm{kV}$ was used to fabricate and bend the cantilevers (Fig. 1). The surroundings of the regions of interest were ion cut and removed, leaving arrays of $5 \times 2 \mu \mathrm{m}^{2}$ cantilevers. This cutting step was performed using an ion current of $\sim 200 \mathrm{pA}$ without any imaging frames to minimize ion irradiation prior to the experiments. Bending experiments were carried using a current of $\sim 40$ pA. A beam overlap of 0.5 (beam diameter $\sim 100 \mathrm{~nm}$ ) was chosen to ensure lateral uniformity. A rectangular area that slightly exceeded the cantilever edges was scanned using a parallel strategy, meaning it was scanned in lines by the ion beam in steps of $50 \mathrm{~nm}$ and dwell time of $1 \mu \mathrm{s}$, repeating for the necessary number of times until the desired fluence was achieved. The cantilevers would then be imaged with SEM. These steps of irradiation and imaging continued up to a maximum $\mathrm{Ga}^{+}$fluence of $2 \times 10^{21} \mathrm{~m}^{-2}$. The deflections were later measured from the SE images using Image [19] and Engauge [20] taking into account the $55^{\circ}$ perspective of the electron beam to the samples normal.

Figure 2 shows a couple of representative SEM images of a set of cantilevers with initial thickness $200 \mathrm{~nm}$, exhibiting initial 'downwards' bending and direction reversal as irradiation progressed. Examples of measured deflection and curvature [explained in the following, with Eq. (19)] for different initial thicknesses are shown in Fig. 3.

The curvature plots show constant values in the cantilever center region, meaning the ion-induced radiation damage is uniform along the cantilever $x$ axis. As fluence increases, the shape of the curvature plots tends to a $U$ shape that can be attributed to the increasingly non-uniform irradiation conditions due to the change in incidence angle toward the cantilever extremity. At the free end, where the deviation from perpendicular incidence is larger, the sputtering yield can be assumed to be larger and the implanted region shallower, giving a heterogeneous deflection along the cantilever length.

The curvature values in the center region were averaged and plotted against fluence as shown in Fig. 4. The deflection response to fluence is more pronounced in the thinner films. The minimum curvature reached by the films with initial thickness 200 and $144 \mathrm{~nm}$ is similar, while the film with $d_{0}=89 \mathrm{~nm}$ showed direction reversal at a smaller negative 


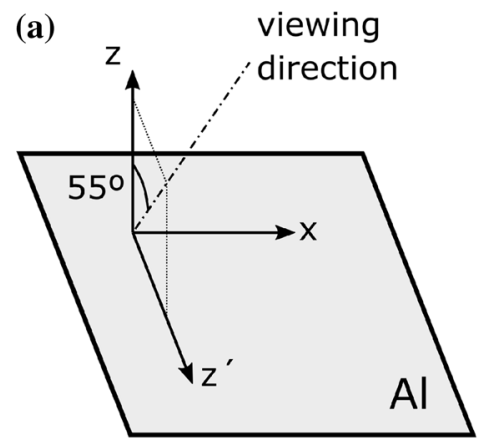

(b)

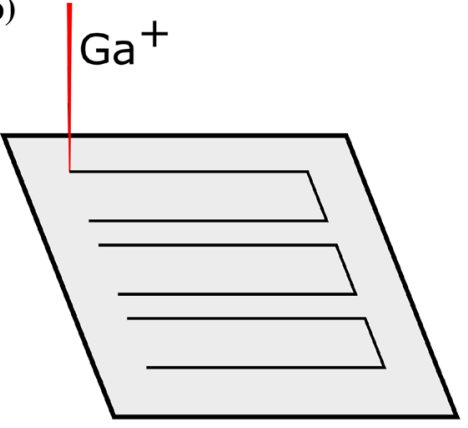

(d)

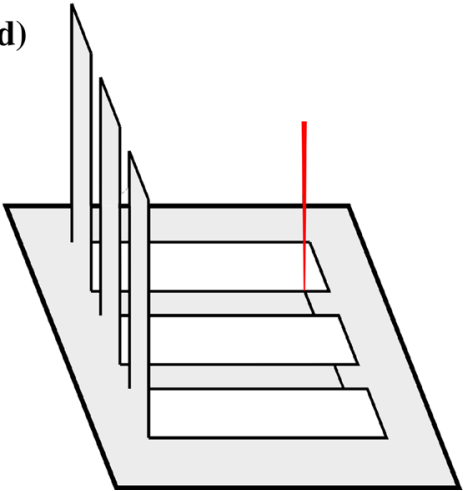

(e)

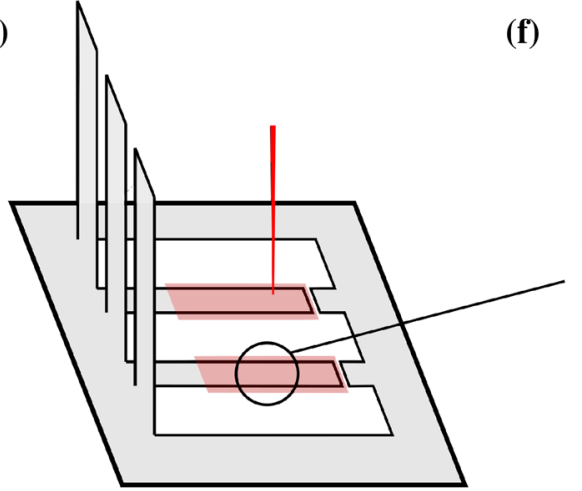

(f) (c)
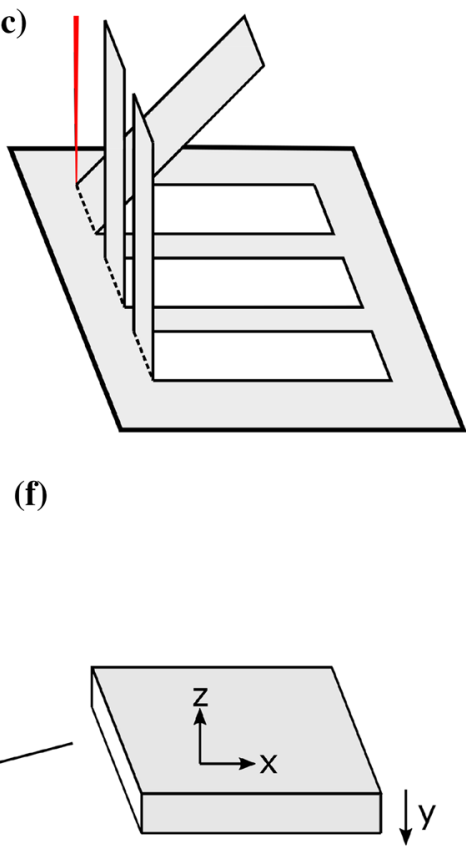

Figure 1 Schematic diagram of cantilevers fabrication and bending: $\mathbf{a}$ free-standing film; $\mathbf{b}$ ion cutting of surrounding areas; c linear irradiation (over the dashed lines) to remove adjacent areas from the line of sight; $\mathbf{d}$ cutting of the cantilevers; e bending experiment: area scan up to desired fluence and subsequent SE imaging; $\mathbf{f} y$-axis is used for modeling defect kinetics in the cantilever as a function of depth, $(x, z)$ is the larger-scale coordinates describing cantilever curvature, Eq. (19).
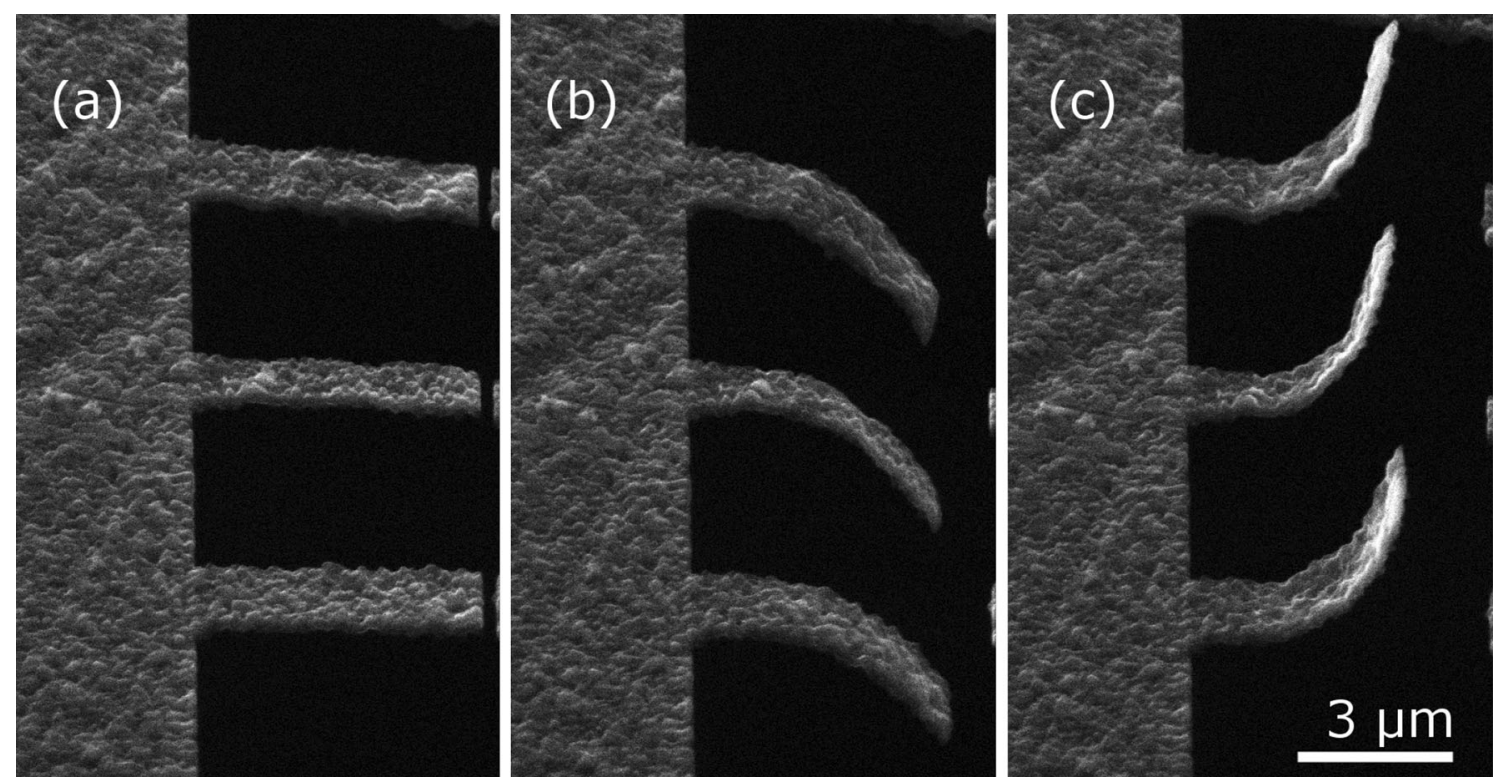

Figure 2 Ion-induced bending of aluminum cantilevers $\left(d_{0}=200 \mathrm{~nm}\right)$ : a initial position; $\mathbf{b}$ deflection after irradiation fluence of $8.0 \times 10^{20} \mathrm{~m}^{-2}$ (when direction reversal took place); and $\mathbf{c}$ deflection after fluence of $1.8 \times 10^{21} \mathrm{~m}^{-2}$.

curvature. This can be reasoned considering the induced swelling distribution width relative to film thickness is larger in that case.
Gallium concentration was measured in a 200-nmthick film for three different irradiation fluences by energy-dispersive X-ray spectroscopy (EDS) in a FEI- 

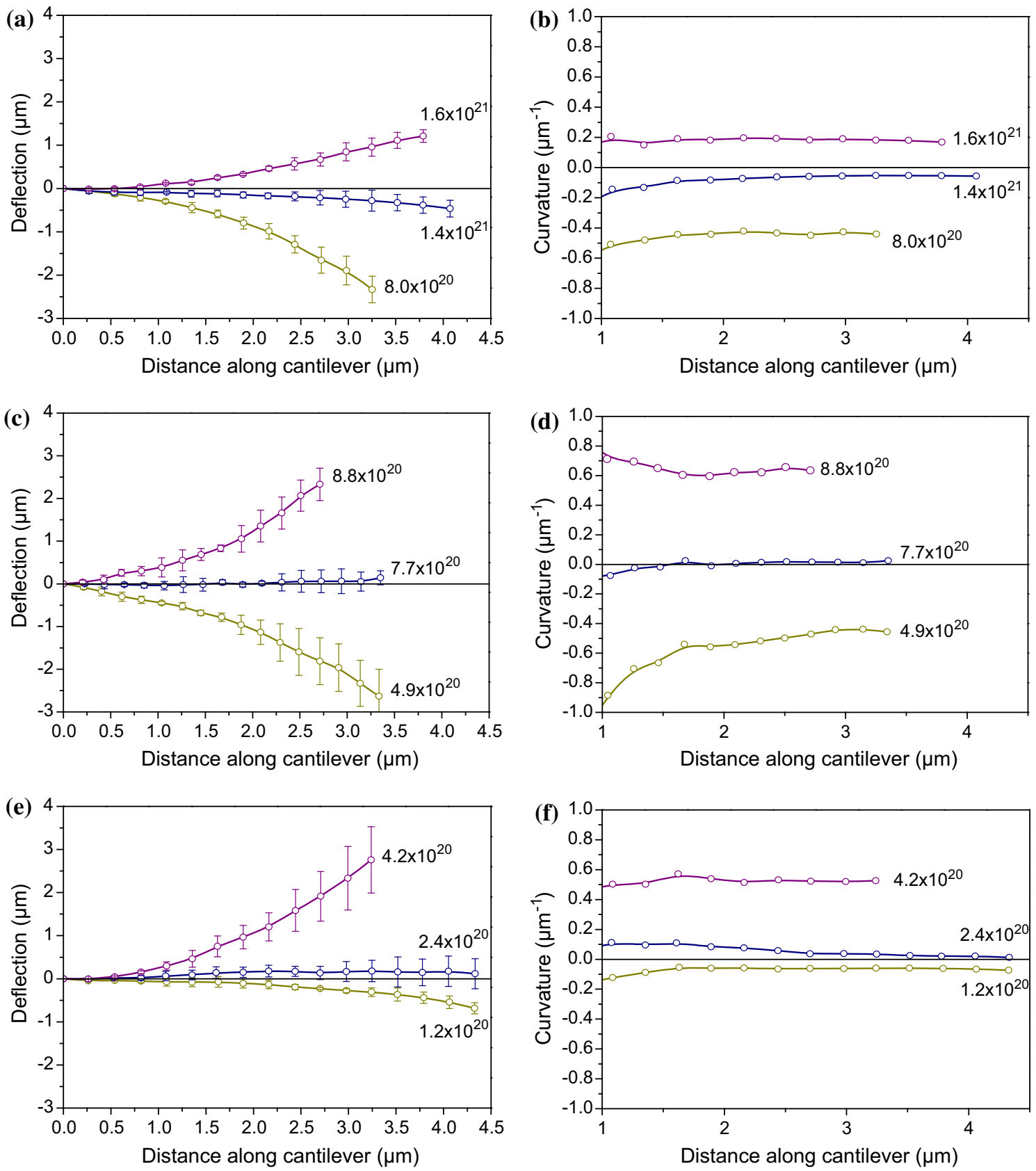

Figure 3 Deflection (left column) and curvature (right column) of cantilever of initial thickness a, b $200 \mathrm{~nm}, \mathbf{c}, \mathbf{d} 144 \mathrm{~nm}$ and e, f $89 \mathrm{~nm}$ irradiated to various fluences indicated in units of $\mathrm{m}^{-2}$. The lines at $y=0$ represent the initial condition.

Philips ESEM XL30F operating at an acceleration voltage of $5 \mathrm{kV}$. The results are shown in Fig. 5 together with the model calculated depth profile and thickness average.

\section{Discussion}

To explain our experimental observations, we formulate a theoretical framework for radiation-induced bending due to radiation damage accumulation in a crystalline thin film. As a $\mathrm{Ga}^{+}$ion travels through the film material, the ion energy dissipates by exciting electrons and by (in)elastic collisions with the material nuclei. Due to collisions some atoms are ejected 


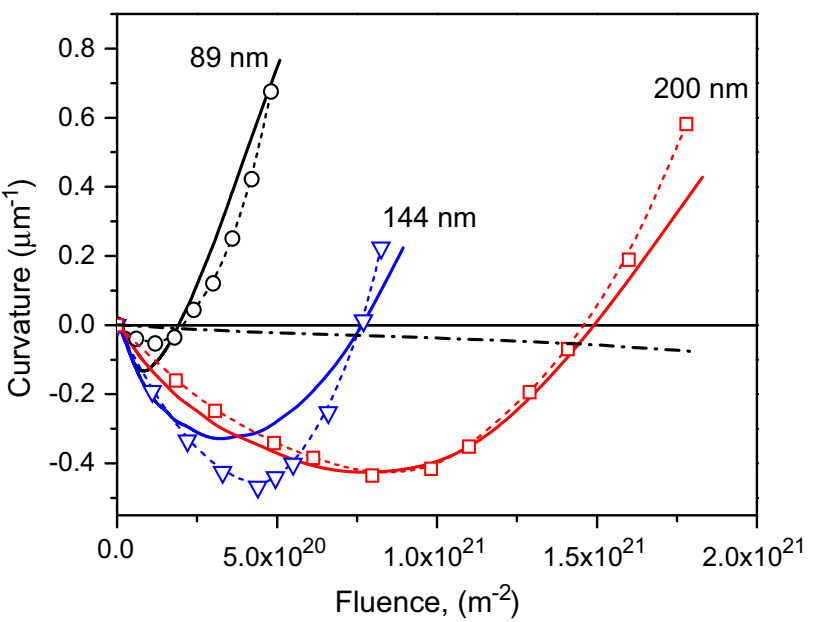

Figure 4 Fluence dependence of the curvature $1 / R$ : comparison of experimental data (symbols) with model calculations (solid lines). The initial thickness of cantilevers is indicated near the corresponding graphs. The negative curvature corresponds to downwards bending. The dashed curve is calculated only with contributions of vacancies and substitutional $\mathrm{Ga}$ atoms to volume change, Eq. (13) at $\varepsilon=0$, when cascades do not produce mobile clusters.

from normal lattice positions creating primary knockon atoms (PKA), which, in turn, may produce cascades of atomic displacements [21, 22]. The defects created in a cascade after a fast relaxation stage include isolated PD (vacancies and interstitial atoms) and small clusters of PD. Point defects continue to migrate by thermal diffusion, resulting, e.g., (1) in the recombination of vacancies with self-interstitials or

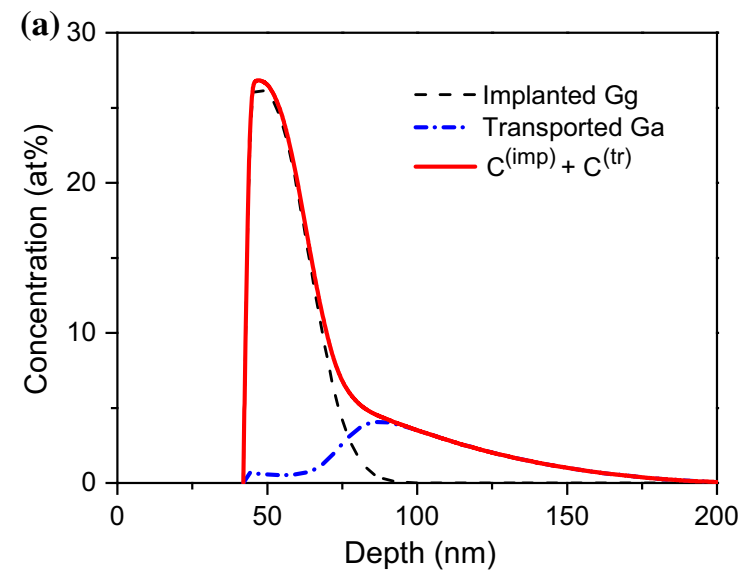

Figure $5 \mathrm{Ga}$ concentration in the $\mathrm{Al}$ film. a The depth dependence at fluence $8 \times 10^{20} \mathrm{~m}^{-2}$. b Fluence dependence of $\mathrm{Ga}$ concentration averaged over film thickness as measured by EDS (symbols) and predicted by the model $\left\langle C_{\mathrm{Ga}}^{(\mathrm{imp})}+C_{\mathrm{Ga}}^{(\mathrm{tr})}\right\rangle$ (solid line); implanted ions and (2) the diffusion of PD to sinks such as surfaces, dislocations, grain boundaries and PD clusters.

According to MD simulations, a significant fraction of the interstitial population is produced in thermally stable clusters, both 'sessile' and 'glissile' [22-24]. Glissile clusters are highly mobile even at room temperature [14] and can migrate away from their parent cascades. In the undamaged region of the film beyond the penetration range of $\mathrm{Ga}^{+}$ions, the mobile clusters can form sessile clusters due to collisions with each other and absorption of single self-interstitial atoms (SIAs). Eventually these clusters may grow into dislocation loops. Migration of interstitial clusters leads to volume increase in the undamaged region since each atom of a glissile cluster brings an excess volume of about the atomic volume, i.e., the relaxation volume of SIA, $\Delta \omega_{i} \sim \omega$. At the same time, there is no accumulation of excess volume in the irradiated subsurface region because cascades create new clusters and destroy existing ones due to cascade overlap and the effect of radiation-induced mixing $[22,25,26]$. In addition, the ion beam sputters the surface atoms and thus removes the damaged subsurface layers. The conclusion is that the ion beam irradiation creates not only PD distributions within the penetration range of ions but results also in material redistribution across the whole thickness of the cantilever. Evolution of material microstructure well below the implantation depth (long-range effect) was observed in various materials [27, 28]. Early

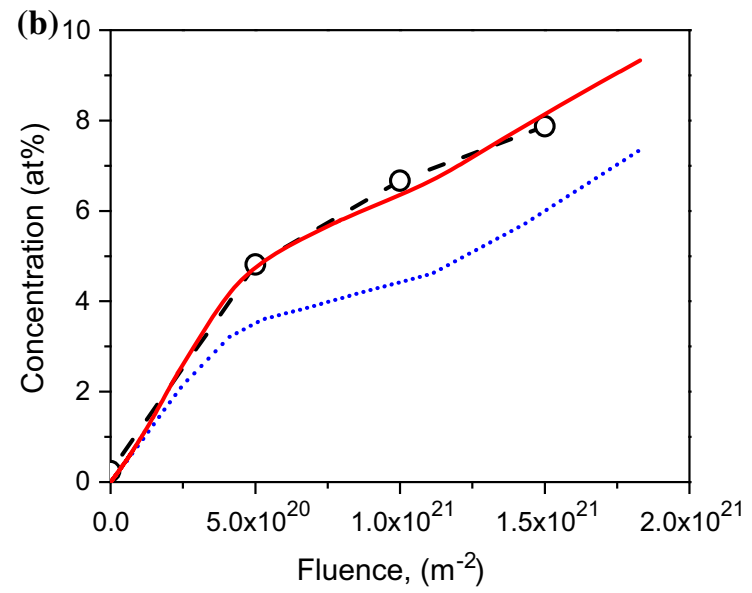

the dotted line corresponds to the average $\mathrm{Ga}$ concentration without contribution of $\mathrm{Ga}$ transported by clusters. The initial thickness is $200 \mathrm{~nm}$; the $\mathrm{Ga}^{+}$flux is $7 \times 10^{18} \mathrm{~m}^{-2} \mathrm{~s}^{-1}$. 
transmission electron microscope (TEM) studies $[29,30]$ on $\mathrm{Cu}$ and Au foils, which were bombarded with 1-5 kV Ar ions, showed that interstitial clusters (in the configuration of Frank sessile dislocation loops) are formed below the bombarded surface at a depth remarkably larger than the calculated random range of Ar ions. A large difference between ion-induced damage depth and theoretically simulated range was observed to be more prominent in fcc metals compared to bcc-Fe [31]. This fact was attributed to a higher value of the Peierls force opposing dislocation glide in bcc structures. Both Rutherford backscattering spectrometry (RBS) channeling spectra and cross-sectional TEM characterization of single crystalline nickel and Ni-based binary alloys irradiated with $3 \mathrm{MV} \mathrm{Au}$ ions clearly demonstrated that the range of radiation-induced defect clusters far exceed the theoretically predicted depth in all materials after high fluence irradiation [27]. The range of defect distribution beneath the irradiated surface increased dramatically with increasing ion fluence. The range of visible damage almost doubled when the irradiation fluence increased from $2 \times 10^{17}$ to $5 \times 10^{19} \mathrm{~m}^{-2}$. The composition of the material was found to have a great impact on defect distribution, suggesting very different defect migration properties. Radiation defects in pure Ni stretched much deeper than in Ni binary alloys, indicating a higher defect migration rate in nickel for both high and low dose irradiation.

It should be noted that according to MD simulation, vacancy clusters (including mobile ones) may also form during early stages of cascade evolution. However, small vacancy clusters and vacancy voids are thermally unstable [22]. For this reason, their effect to bending is expected to be small as compared to interstitial clusters.

To estimate the contribution of PD and interstitial clusters to cantilever bending, the analysis presented above is used to formulate of radiation damage evolution in a thin film.

Let us consider a cantilever beam of thickness $d_{0}$ irradiated with $\mathrm{Ga}^{+}$ions. The primary defects produced by displacement cascades are isolated PD and mobile interstitial clusters. For simplicity, we assume that clusters are of the same size, $m$. Doing this has the major advantage that it allows to reduce the number of fitting parameters. Mobile clusters undergo random walks and form sessile clusters when collide with each other. We do not follow subsequent evolution of sessile clusters. In this study the most important property of sessile clusters is their volume as a function of depth. The time-dependent rate equations for concentrations of vacancies $C_{v}$, selfinterstitial atoms $C_{\mathrm{i}}$, interstitial $\mathrm{Ga}$ atoms $C_{\mathrm{iGa}}$, substitutional $\mathrm{Ga}$ atoms $C_{\mathrm{Ga}}$, mobile clusters $C_{\mathrm{m}}$ and sessile clusters $C_{\mathrm{S}}$ are as follows

$\frac{\mathrm{d} C_{\mathrm{v}}}{\mathrm{d} t}=K\left(1-C_{\mathrm{v}}\right)-\alpha C_{\mathrm{v}}\left(D_{\mathrm{i}} C_{\mathrm{i}}+D_{\mathrm{iGa}} C_{\mathrm{iGa}}\right)+D_{\mathrm{v}} \Delta C_{\mathrm{v}}$

$\frac{\mathrm{d} C_{\mathrm{i}}}{\mathrm{d} t}=K(1-\varepsilon)\left(1-C_{\mathrm{v}}-C_{\mathrm{Ga}}\right)+\eta K m\left(C_{\mathrm{m}}+2 C_{\mathrm{S}}\right)$ $-\alpha D_{\mathrm{i}} C_{\mathrm{i}} C_{\mathrm{v}}+D_{\mathrm{i}} \Delta C_{\mathrm{i}}$,

$\frac{\mathrm{d} C_{\mathrm{iGa}}}{\mathrm{d} t}=K_{\mathrm{Ga}}+K(1-\varepsilon) C_{\mathrm{Ga}}-\alpha D_{\mathrm{iGa}} C_{\mathrm{iGa}} C_{\mathrm{v}}+D_{\mathrm{iGa}} \Delta C_{\mathrm{iGa}}$,

$\frac{\mathrm{d} C_{\mathrm{Ga}}}{\mathrm{d} t}=\alpha D_{\mathrm{iGa}} C_{\mathrm{iGa}} C_{\mathrm{v}}-K(1-\varepsilon) C_{\mathrm{Ga}}$,

$\frac{\mathrm{d} C_{\mathrm{m}}}{\mathrm{d} t}=\frac{\varepsilon K}{m}\left(1-C_{\mathrm{v}}\right)-\eta K C_{\mathrm{m}}-2 \alpha_{\mathrm{m}} D_{\mathrm{m}} C_{\mathrm{m}}^{2}+D_{\mathrm{m}} \Delta C_{\mathrm{m}}$,

$\frac{\mathrm{d} C_{\mathrm{S}}}{\mathrm{d} t}=\alpha_{\mathrm{m}} D_{\mathrm{m}} C_{\mathrm{m}}^{2}-\eta K C_{\mathrm{S}}$,

where concentrations are defined per lattice site, $K$ is the depth-dependent generation rate of $\mathrm{PD}, \varepsilon$ is the fraction of clustered interstitial atoms, $\eta=\beta V_{\mathrm{dc}} / \omega n_{\mathrm{d}}$ is a cluster destruction parameter (see explanation below), $\beta$ is probability to destroy a cluster by a cascade, $V_{\mathrm{dc}}$ is volume of a displacement cascade, $\omega$ is the volume per lattice site, $n_{\mathrm{d}}$ is the number of defects of one type produced in a cascade, $K_{\mathrm{Ga}}$ is the implantation rate of $\mathrm{Ga}, \alpha=4 \pi R_{\mathrm{iv}} / \omega$ is the recombination rate constant, $R_{\mathrm{iv}} \sim a$ is the radius of spontaneous recombination ( $a$ is the lattice spacing), $D_{\mathrm{i}, \mathrm{v}, \mathrm{iGa}, \mathrm{m}}$ are the diffusion coefficients $\left(D_{\mathrm{v}}<D_{\mathrm{m}}<D_{\mathrm{i}, \mathrm{GGa}}\right)$. For simplicity, diffusion coefficient of self-interstitial atoms and interstitial Ga ions are assumed to be the same. The rate constant for cluster formation is written in the so-called Smoluchowski approximation [32]

$\alpha_{\mathrm{m}}=\frac{8 \pi}{\omega} d_{\mathrm{m}} D_{\mathrm{m}}, \quad d_{\mathrm{m}}=2\left(\frac{3 m \omega}{4 \pi}\right)^{1 / 3}$,

where $d_{\mathrm{m}}$ is the effective cluster diameter.

The PD generation rate and Ga implantation rate per second and per lattice site are calculated with SRIM [33] 
$K(y)=j \omega K_{\text {SRIM }}(y)$,

$K_{\mathrm{Ga}}(y)=j \omega K_{\mathrm{SRIM}}^{\mathrm{Ga}}(y)$,

where $j$ is the flux of $\mathrm{Ga}^{+}$ions at the film surface, $K_{\text {SRIM }}(y)$ is the distribution of vacancies over the film depth $y$ per incident $\mathrm{Ga}^{+}$ion, and $K_{\text {SRIM }}^{\mathrm{Ga}}(y)$ is the probability density to find the incident ion at a depth $y$ when it stops.

The parameter $\eta K=\beta K V_{\mathrm{dc}} / \omega n_{\mathrm{d}}$ is the inverse lifetime of a cluster due to ballistic and thermal spike effects $[22,25,26]$. Here $K / \omega n_{d}$ is the generation rate of cascades per second per unit volume. It is assumed that if a cascade of volume $V_{\mathrm{dc}}$ develops in the neighborhood of a cluster, then it destroys a cluster with the probability $\beta<1$.

The impingement of the film with energetic ions results in film sputtering, i.e., movement or receding of the film boundary with the rate

$v=\frac{\mathrm{d} y}{\mathrm{~d} t}=j \omega Y$,

where $Y$ is the sputtering yield (number of sputtered atoms per incident ion).

For PD in Eqs. (1)-(3) we use zero boundary conditions at both external surfaces.

$\left.\frac{\mathrm{d} C_{\mathrm{v}, \mathrm{i}, \mathrm{iGa}}}{\mathrm{d} y}\right|_{y=v t}=0,\left.\quad \frac{\mathrm{d} C_{\mathrm{v}, \mathrm{i}, \mathrm{iGa}}}{\mathrm{d} y}\right|_{y=d_{0}}=0$.

For mobile clusters, we use mixed boundary conditions,

$\left.\frac{\mathrm{d} C_{\mathrm{m}}}{\mathrm{d} y}\right|_{y=v t}=-\left.\gamma C_{\mathrm{m}}\right|_{y=v t},\left.\quad \frac{\mathrm{d} C_{\mathrm{m}}}{\mathrm{d} y}\right|_{y=d_{0}}=-\left.\gamma C_{\mathrm{m}}\right|_{y=d_{0}}$,

assuming that the flux of clusters at the external surfaces is proportional to cluster concentration near surfaces, i.e., the surface is not a perfect sink for clusters as distinct from PD. A physical interpretation of this assumption is related to how fast interstitial clusters are transformed to be absorbed by external surfaces.

PD can be absorbed by dislocations and grain boundaries. However, it is assumed in the model that the material is well annealed; i.e., the sink strength of dislocations and grain boundaries is low as compared to sink strength of external surfaces. This means that the film thickness is smaller than the mean distance between grain boundaries and the dislocation spacing.

From a physics viewpoint, the bending is a consequence of the stress originating from the inhomogeneous volume change due to implanted ions, PD distributions and defect clusters. The relative volume change $\Omega=\Delta V / V$ associated with implanted ion and PD is given by the summation:

$\Omega_{\mathrm{PD}}=C_{\mathrm{v}}(y) \frac{\Delta \omega_{\mathrm{v}}}{\omega}+C_{\mathrm{Ga}}(y) \frac{\Delta \omega_{\mathrm{Ga}}}{\omega}$,

where $\Delta \omega_{\mathrm{v}}$ is the vacancy relaxation volume and $\Delta \omega_{\mathrm{Ga}}$ is the excess volume associated with substitutional $\mathrm{Ga}$ atom. Here we neglected the contribution of isolated interstitial atoms because the remaining concentrations of interstitial atoms are very small due to high diffusion mobility at room temperature.

The relaxation volume of vacancies is negative in all metals. The excess volume associated with substitutional $\mathrm{Ga}$ atom depends on the specific film material, being positive in $\mathrm{Al}$ (Table 1). It can be deduced from the dependence of lattice parameter on Ga concentration in solid solutions [34].

Another contribution to the volume change originates from interstitial clusters

$\Omega_{\mathrm{mS}}=m\left(C_{\mathrm{m}}+2 C_{\mathrm{S}}\right) \Delta \omega_{\mathrm{i}} / \omega$.

To evaluate the bending due to the inhomogeneous volume change

$\Omega=\Omega_{\mathrm{PD}}+\Omega_{\mathrm{mS}}$

we use an analogy with the thermoelasticity. It is well known that thermoelastic stresses due to the linear thermal expansion $\alpha T(y)$ results in bending of beams and multilayered materials [35]. In the equations of thermoelastic bending of the cantilever beam, we replace the linear thermal expansion $\alpha T(y)$ with the linear expansion/contraction

$\lambda(y)=\Omega(y) / 3$

and find the solution to the problem of cantilever bending induced by ion irradiation. The strain in the film is given by

$$
\begin{gathered}
\varepsilon_{x x}=\varepsilon_{0}+\frac{1}{R}\left(y-\frac{d}{2}\right), \quad \varepsilon_{0}=\frac{1}{d} \int_{0}^{d} \lambda(y) \mathrm{d} y, \\
\frac{1}{R}=\frac{12}{d^{3}} \int_{0}^{d} \lambda(y)\left(y-\frac{d}{2}\right) \mathrm{d} y
\end{gathered}
$$

where $d=d_{0}-v t$ is the cantilever thickness, $x$ is the coordinate in the direction of the free end, $R$ is the radius of curvature at the neutral plane and $\varepsilon_{0}$ is the axial strain in the $x$-direction at the neutral plane. The stress in the cantilever is given by 
Table 1 Material parameters used in model calculations

\begin{tabular}{ll}
\hline Parameter & Value \\
\hline Flux of $\mathrm{Ga}^{+}$ions $j\left(\mathrm{~m}^{-2} \mathrm{~s}^{-1}\right)$ & $3 \times 10^{19}$ \\
Temperature, $T(\mathrm{~K})$ & 300 \\
Sputtering yield, $Y, \mathrm{SRIM}$ & 3.8 \\
Recombination rate constant, $\alpha\left(\mathrm{m}^{-2}\right)$ & $4.8 \times 10^{20}$ \\
Rate constant for cluster formation, $\alpha_{\mathrm{m}}\left(\mathrm{m}^{-2}\right)$ & $7.6 \times 10^{20}$ \\
Diffusion coefficient of vacancies, $D_{\mathrm{v}}\left(\mathrm{m}^{2} \mathrm{~s}^{-1}\right)$ & $7 \times 10^{-6} \exp \left(-0.62 \mathrm{eV} / \mathrm{k}_{\mathrm{B}} T\right)$ \\
Diffusion coefficient of interstitials, $D_{\mathrm{i}}\left(\mathrm{m}^{2} \mathrm{~s}^{-1}\right)$ & $5 \times 10^{-6} \mathrm{exp}\left(-0.1 \mathrm{eV} / \mathrm{k}_{\mathrm{B}} T\right)$ \\
Diffusion coefficient of clusters, $D_{\mathrm{m}}\left(\mathrm{m}^{2} \mathrm{~s}^{-1}\right)$ & $10^{-5} \exp \left(-0.3 \mathrm{eV} / k_{\mathrm{B}} T\right)$ \\
Atomic volume, $\omega$ (m $\left.{ }^{-3}\right)$ & $1.66 \times 10^{-29}$ \\
Relaxation volume of vacancies, $\Delta \omega_{\mathrm{v}}$ & $-0.33 \omega$ \\
Relaxation volume of interstitial atoms, $\Delta \omega_{\mathrm{i}}$ & $\approx \omega$ \\
Misfit volume of gallium, $\Delta \omega_{\mathrm{Ga}}$ & $0.08 \omega$ \\
Number of atoms in cluster, $m$ & 4 \\
Fraction interstitial atoms produced in clusters, $\varepsilon$ & 0.1 \\
Destruction parameter, $\eta$ & 0.2 \\
Parameter $\gamma\left(\mathrm{nm}{ }^{-1}\right)$ & 0.06 \\
\hline
\end{tabular}

$\frac{\sigma_{x x}}{E}=\varepsilon_{0}+\frac{1}{R}\left(y-\frac{d}{2}\right)-\lambda(y)$

where $E$ is the Young's modulus. The deflection of the beam is related to curvature by

$z=x^{2} / 2 R$

The bending direction depends on the asymmetry of excess volume distribution with respect to the neutral axis. It should be noted that the curvature radius, Eq. (17), depends only on the inhomogeneous volume change. One may expect that the volume change can generate high stresses leading to plastic deformation.

In this work the set of equations formulated above was solved numerically by the method of lines using the RADAU code [36]. Material parameters used in simulations are listed in Table 1.

The cluster diffusion coefficient $D_{\mathrm{m}}$ and parameters $\varepsilon, \eta$ and $\gamma$ were adjusted to reproduce experimental behavior of the cantilever curvature. In our model the cluster migration energy is $0.3 \mathrm{eV}$, i.e., higher than reported by $\mathrm{MD}$ simulations for $1 \mathrm{D}$ migration $[14,24]$. The difference can be explained that in our case the coefficient $D_{\mathrm{m}}$ is the effective coefficient of 3D diffusion, which requires cluster reorientation.

The production rate of PD $K$ and the implantation rate of $\mathrm{Ga} K_{\mathrm{Ga}}$ were calculated with SRIM for $30 \mathrm{keV}$ Ga ion implanted into amorphous $\mathrm{Al}$ target at normal incidence. The 'monolayer collisions-surface sputtering' option of SRIM was selected with displacement energy $25 \mathrm{eV}$ [37] (Fig. 6). According to recommendations [38] the SRIM data for number of defects produced by an ion were divided by factor of 2 , since SRIM overestimate number of displaced atoms as compared to more realistic MD simulations. SRIM simulations predict that during irradiation of $\mathrm{Al}$ film with $30 \mathrm{kV}$ Ga ions, PD are generated mostly within a range of about $50 \mathrm{~nm}$.

For a cantilever of initial thickness $200 \mathrm{~nm}$, the concentration profiles of vacancies and substitutional $\mathrm{Ga}$ atoms are shown in Fig. 7. It is seen that the concentration profiles move inside the film as the material is removed from the surface due to sputtering. Concentrations of SIAs and Ga interstitial atoms are less than $10^{-12}$ because of fast diffusion to external surfaces. At the simulation temperature, the

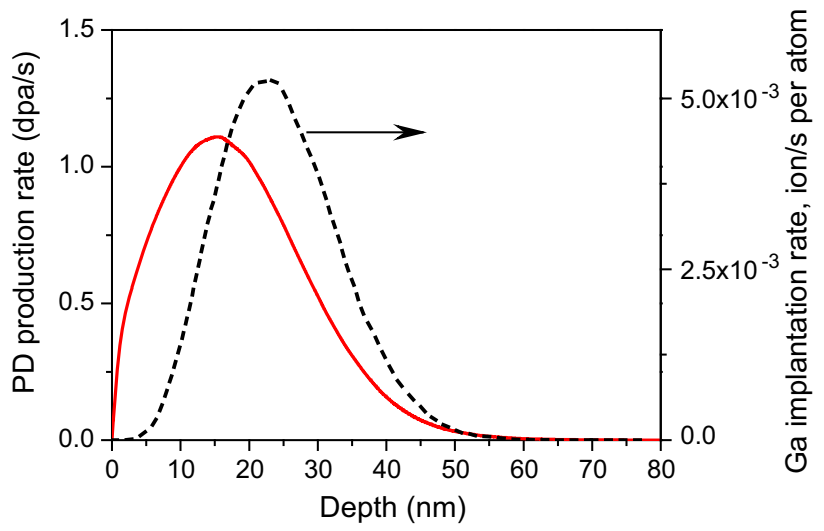

Figure $6 \mathrm{PD}$ production rate $K$ and $\mathrm{Ga}$ implantation rate $K_{\mathrm{Ga}}$ calculated with SRIM for $30 \mathrm{keV} \mathrm{Ga}^{+}$ion implanted into amorphous $\mathrm{Al}$ target at normal incidence. The $\mathrm{Ga}^{+}$flux is $7 \times 10^{18} \mathrm{~m}^{-2} \mathrm{~s}^{-1}$. 
(a)

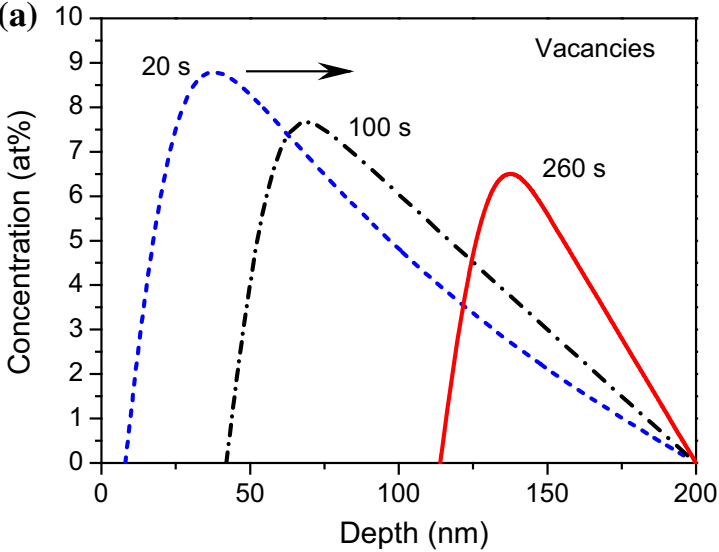

(b)

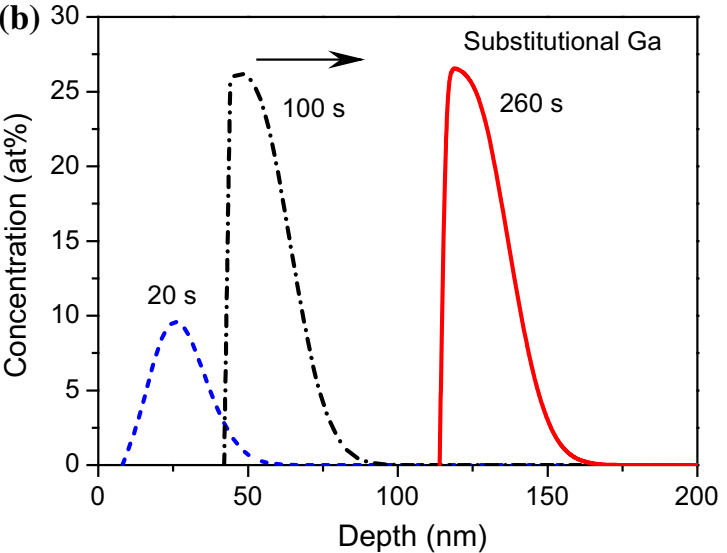

Figure 7 Concentration profiles of vacancies (a) and implanted Ga atoms (b). The initial thickness is $200 \mathrm{~nm}, \mathrm{fhe}^{+}$flux is $7 \times 10^{18} \mathrm{~m}^{-2} \mathrm{~s}^{-1}$. The left boundary of the film is moving to the right (indicated with arrow) because of sputtering.

timescale of vacancy diffusion is $(100 \mathrm{~nm})^{2} / D_{\mathrm{v}}=37 \mathrm{~s}$. It should be noted that distributions of $\mathrm{Ga}$ atoms in the subsurface region of thickness $50 \mathrm{~nm}$ do not depend on time after a short transient period.

Figure 8 shows concentration profiles of mobile and sessile clusters. It seen that the sessile clusters are destroyed near the film surface exposed to the ion beam. As the surface moves away from the beam, concentration of sessile clusters increases in the region beyond the penetration range of ions.

Accumulation of radiation defects results in a high swelling of material, up to $40 \%$ in the maximum (Fig. 9). Vacancies that escaped recombination with interstitials are removed from the system because of sputtering. Clustered interstitial atoms survive in the form of sessile clusters, which evolve into dislocation loops, giving rise to swelling of the film. Up to a fluence of $8 \times 10^{20} \mathrm{~m}^{-2}(114 \mathrm{~s})$ the $200 \mathrm{~nm}$ cantilever deflects away from the beam; the curvature is negative (Fig. 4). The reason is that most of the swelling occurs between the irradiated surface and the neutral axis (Fig. 9). As the film becomes thinner, the bending direction changes.

Our theoretical predictions agree well with the experimental dependence of curvature on fluence and initial cantilever thickness (Fig. 4). The cluster mechanism of bending is supported by our observation that bending cannot be removed by annealing, whereas bending due to isolated PD would anneal completely.

Figure 4 shows also the fluence dependence of bending at $\varepsilon=0$, i.e., in a system without production of clusters. In this case the negative value of curvature is due to positive volume misfit of substitutional Ga atoms which dominates vacancy contribution. It is seen that distributions of isolated PD defect in the
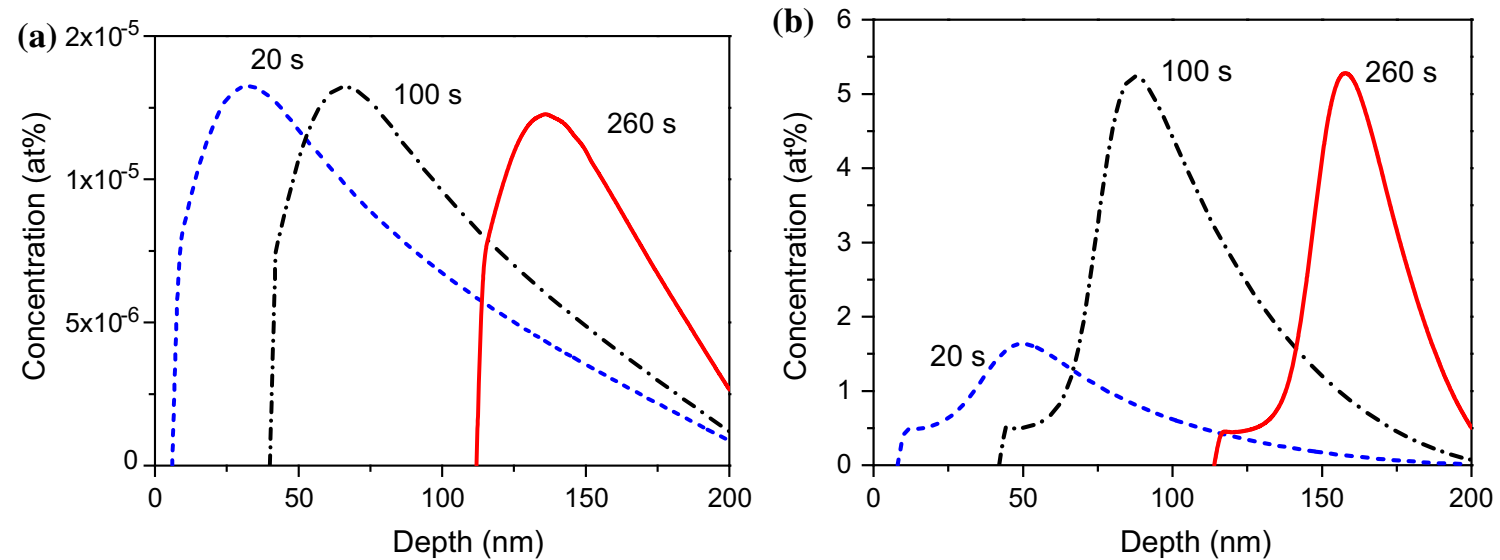

Figure 8 Concentration profiles of mobile (a) and sessile clusters (b). The initial thickness is $200 \mathrm{~nm}$; the Ga $\mathrm{Gax}^{+}$is $7 \times 10^{18} \mathrm{~m}^{-2} \mathrm{~s}^{-1}$. 


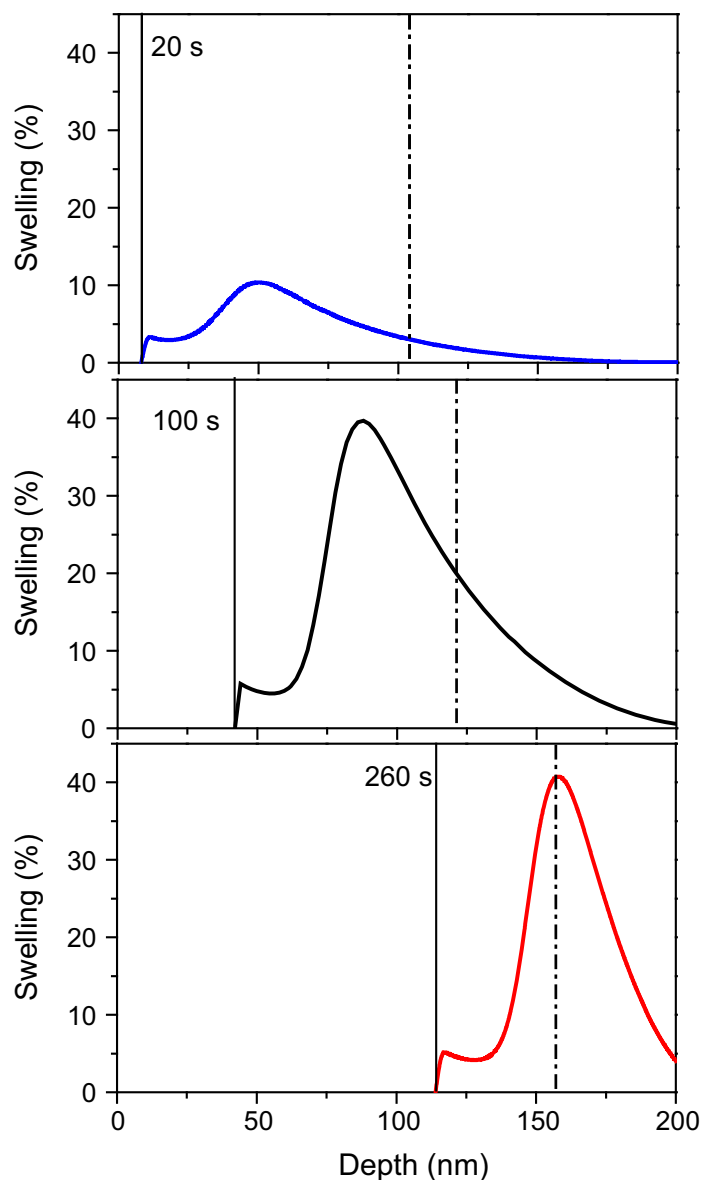

Figure 9 Swelling $\Omega$ as a function of depth. The initial thickness is $200 \mathrm{~nm}$; the $\mathrm{Ga}^{+}$flux is $7 \times 10^{18} \mathrm{~m}^{-2} \mathrm{~s}^{-1}$. The vertical solid lines show the position of the film left boundary subjected to ion irradiation. The dash-dotted lines show the center lines of the film (neutral axis).

damaged region cannot produce the observed cantilever curvature.

Note that mobile interstitial clusters, which form during cascade evolution, can transport Ga atoms to the unirradiated region. Assuming that the probability to find $\mathrm{Ga}$ atoms in clusters equals the $\mathrm{Ga}$ concentration averaged over the implantation profile thickness $\left\langle C_{\mathrm{Ga}}^{(\mathrm{imp})}\right\rangle$ (see Fig. $7 \mathrm{~b}$ ), the increment of $\mathrm{Ga}$ concentration in this region during small time interval is written as

$\mathrm{d} C_{\mathrm{Ga}}^{(\mathrm{tr})}=\left\langle C_{\mathrm{Ga}}^{(\mathrm{imp})}\right\rangle \frac{\mathrm{d} \Omega_{\mathrm{mS}}}{1+\Omega_{\mathrm{mS}}}$,

Since the Ga implantation profile practically do not change after a short transient period, i.e., $\left\langle C_{\mathrm{Ga}}^{(\mathrm{imp})}\right\rangle \approx$ const, Eq. (20) is easily integrated. The total $\mathrm{Ga}$ concentration in the film is given by

$C_{\mathrm{Ga}}=C_{\mathrm{Ga}}^{(\mathrm{imp})}+C_{\mathrm{Ga}}^{(\mathrm{tr})}=C_{\mathrm{Ga}}^{(\mathrm{imp})}+\left\langle C_{\mathrm{Ga}}^{(\mathrm{imp})}\right\rangle \ln \left(1+\Omega_{\mathrm{mS}}\right)$

Figure 5 compares model estimations with EDS measurements of $\mathrm{Ga}$ concentration in $200 \mathrm{~nm} \mathrm{Al}$ cantilever. The model predicts that $\mathrm{Ga}$ can be found beyond the implantation peak.

To summarize the discussion, we would like to note that we are aware of the assumptions and simplifications, but the essentials are validated and supported by experimental observations. Our goal was (1) to highlight the important role of radiation damage beyond of implantation range of $\mathrm{Ga}$ ions and (2) to demonstrate that bending is the response of thin cantilevers to essential microstructural changes and material redistribution resulting in high swelling.

\section{Conclusions}

A series of bending experiments with cantilevers made from $\mathrm{Al}$ was performed. The same surprising behavior was observed in cantilevers of various thickness: all samples bent initially away from the $30 \mathrm{kV} \mathrm{Ga}^{+}$beam and reversed the bending direction as irradiation proceeded. The experimental data are discussed in terms of local volume change due to mass transfer from the cascade region to the so-called undamaged region. To this end the model for defect diffusion kinetics in the crystalline thin films under irradiation with energetic ions has been formulated. An analogy with thermoelasticity is used to convert the inhomogeneous volume change along the thickness of a free-standing cantilever into bending curvature.

An important and novel message of the work is that the bending under ion beam irradiation cannot be explained by the generation of isolated PD and deposition of $\mathrm{Ga}$ ions since their effects are too small. Our experimental findings are consistent with the mechanism of gliding/diffusion of interstitial clusters, which eventually form sessile clusters. The amount of material transferred to the unirradiated zone grows with the fluence and leads to swelling in regions well beyond the penetration range of $\mathrm{Ga}$ ions. The proposed model predicts that the range of ioninduced microstructural changes exceeds far the 
SRIM predicted implantation depth. It is concluded that a predictive and useful model was presented for the fabrication of micro- and nano-sized objects.

\section{Acknowledgements}

CAPES Foundation, Ministry of Education of Brazil, Brasília-DF 70040-020, Brazil \{99999.000578/2014$02\}$ is gratefully acknowledged for financial support of D.R.G. The Netherlands Organization for Scientific Research NWO \{Grant \# 040.11.511\} is acknowledged for awarding a visitor's Grant to A.A.T.

\section{Compliance with ethical standards}

Conflicts of interest The authors declare that they have no conflicts of interest.

Open Access This article is distributed under the terms of the Creative Commons Attribution 4.0 International License (http://creativecommons.org/ licenses/by/4.0/), which permits unrestricted use, distribution, and reproduction in any medium, provided you give appropriate credit to the original author(s) and the source, provide a link to the Creative Commons license, and indicate if changes were made.

\section{References}

[1] Sugiyama M, Sigesato G (2004) A review of focused ion beam technology and its applications in transmission electron microscopy. J Electron Microsc (Tokyo) 53:527-536

[2] Schaffer M, Schaffer B, Ramasse Q (2012) Sample preparation for atomic-resolution STEM at low voltages by FIB. Ultramicroscopy 114:62-71

[3] Focused Ion Beam Technology and Applications (2014) MRS Bulletin 39(4):317-365

[4] Wang ZM (ed) (2014) FIB nanostructures. Springer, New York

[5] Park BC, Jung KY, Song WY, B-h O, Ahn SJ (2006) Bending of a carbon nanotube in vacuum using a focused ion beam. Adv Mater 18:95-98

[6] Rajput NS, Tong Z, Luo X (2015) Investigation of ion induced bending mechanism for nanostructures. Mater Res Express 2:015002

[7] Camara O, Hanif I, Tunes M, Harrison R, Greaves G, Donnelly S, Hinks J (2017) Effects of temperature on the ion-induced bending of germanium and silicon nanowires. Mater Res Express 4:075056

[8] Bettge M, MacLaren S, Burdin S, Haasch RT, Abraham D, Petrov I, Yu M-F, Sammann E (2012) Ion-induced surface relaxation: controlled bending and alignment of nanowire arrays. Nanotechnology 23:175302

[9] Kozeki T, Phan HP, Dao DV, Inoue S, Namazu T (2016) Influence of gallium ion beam acceleration voltage on the bend angle of amorphous silicon cantilevers. Jpn J Appl Phys 55:06GL02

[10] Punzhin S, Detsi E, Kuzmin A, De Hosson JTM (2014) Deformation of nanoporous nanopillars by ion beam-induced bending. J Mater Sci 49:5598-5605. https://doi.org/ 10.1007/s10853-014-8269-1

[11] Yoshida T, Nagao M, Kanemaru S (2009) Development of thin-film bending technique induced by ion-beam irradiation. Appl Phys Express 2:066501

[12] Arora WJ, Sijbrandij S, Stern L, Notte J, Smith HI, Barbastathis G (2007) Membrane folding by helium ion implantation for three-dimensional device fabrication. J Vac Sci Technol B 25:2184-2187

[13] Rajput NS, Banerjee A, Verma HC (2011) Electron- and ionbeam-induced maneuvering of nanostructures: phenomenon and applications. Nanotechnology 22:485302

[14] Bacon DJ, Osetsky YN (2004) Multiscale modelling of radiation damage in metals: from defect generation to material properties. Mater Sci Eng A 365:46-56

[15] Calder AF, Bacon DJ, Barashev AV, Osetsky YN (2010) On the origin of large interstitial clusters in displacement cascades. Philos Mag 90:863-884

[16] Hofmann F, Tarleton E, Harder RJ, Phillips NW, Ma P-W, Clark JN, Robinson IK, Abbey B, Liu W, Beck CE (2017) 3D lattice distortions and defect structures in ion-implanted nano-crystals. Sci Rep 7:45993

[17] Idrissi H, Turner S, Mitsuhara M, Wang B, Hata S, Coulombier M, Raskin J-P, Pardoen T, Van Tendeloo G, Schryvers D (2011) Point defect clusters and dislocations in FIB irradiated nanocrystalline aluminum films: an electron tomography and aberration-corrected high-resolution ADFSTEM study. Microsc Microanal 17:983-990

[18] Lee S, Jeong J, Kim Y, Han SM, Kiener D, Oh SH (2016) FIB-induced dislocations in al submicron pillars: annihilation by thermal annealing and effects on deformation behavior. Acta Mater 110:283-294

[19] Schneider CA, Rasband WS, Eliceiri KW (2012) NIH Image to ImageJ: 25 years of image analysis. Nat Methods 9:671-675

[20] Mitchell M, Muftakhidinov B, Winchen T, JędrzejewskiSzmek Z, Badger TG (2016) engauge-digitizer: Version 9.2 
Bug fix release https://zenodo.org/record/61028\#. WSwGJuuGO70

[21] Bacon DJ, Osetsky YN, Stoller R, Voskoboinikov RE (2003) MD description of damage production in displacement cascades in copper and $\alpha$-iron. J Nucl Mater 323:152-162

[22] Was GS (2007) Fundamentals of radiation materials science-metals and alloys. Springer, Berlin

[23] Bacon DJ, Gao F, Osetsky YN (2000) The primary damage state in fcc bcc and hcp metals as seen in molecular dynamics simulations. J Nucl Mater 276:1-12

[24] Terentyev DA, Malerba L, Hou M (2007) Dimensionality of interstitial cluster motion in bcc-Fe. Phys Rev B 75:104108

[25] Naundorf V (1992) Diffusion in metals and alloys under irradiation. Int J Mod Phys B 06:2925-2986

[26] Turkin AA, Abromeit C, Naundorf V (1997) Modeling of low temperature transport due to radiation-induced atomic mixing. Defect Diffus Forum 143-147:521-528

[27] Lu C, Jin K, Béland LK, Zhang F, Yang T, Qiao L, Zhang Y, Bei H, Christen HM, Stoller RE, Wang L (2016) Direct observation of defect range and evolution in ion-irradiated single crystalline Ni and Ni binary alloys. Sci Rep 6:19994

[28] Kiener D, Motz C, Rester M, Jenko M, Dehm G (2007) FIB damage of $\mathrm{cu}$ and possible consequences for miniaturized mechanical tests. Mater Sci Eng A 459:262-272

[29] Diepers H, Diehl J (1966) Nachweis und entstehung von zwischengitteratom-agglomeraten in ionenbestrahlten kupferfolien. Phys Status Solidi B 16:K109-K112

[30] Hertel B, Diehl J, Gotthardt R, Sultze H (1974) Formation of interstitial agglomerates and gas bubbles in cubic metals irradiated with $5 \mathrm{keV}$ argon ions. In: Picraux ST, EerNisse
EP, Vook FL (eds) Applications of ion beams to metals. Springer, Boston, pp 507-520. https://doi.org/10.1007/9781-4684-2079-1_40

[31] Friedland E, Alberts HW (1988) Deep radiation damage in metals after ion implantation. Nucl Instrum Methods Phys Res B 33:710-713

[32] Smoluchowski M (1917) Versuch einer mathematischen theorie der koagulationskinetik kolloider losungen. Z Phys Chem 92:129-168

[33] Ziegler JF, Ziegler MD, Biersack JP (2010) SRIM-the stopping and range of ions in matter. Nucl Instrum Meth Phys Res Sect B 268:1818-1823

[34] Predel B, Stein D-W (1969) Konstitution und thermodynamische eigenschaften der legierungen des systems aluminium-gallium. J Less-Common Met 17:377-390

[35] Hetnarski RB, Eslami MR (2009) Thermal stresses-advanced theory and applications. Springer, New York, pp 219-252

[36] Hairer E, Wanner G (1996) Solving ordinary differential equations II Stiff and differential-algebraic problems, vol 14. Springer Series in Comput Mathematics. Springer, Berlin

[37] ASTM E521-16 (2016) Standard practice for investigating the effects of neutron radiation damage using charged-particle irradiation. ASTM International West Conshohocken PA. https://www.astm.org/Standards/E521.htm

[38] Stoller RE, Toloczko MB, Was GS, Certain AG, Dwaraknath S, Garner FA (2013) On the use of SRIM for computing radiation damage exposure. Nucl Instrum Methods Phys Res B 310:75-80 